\title{
Editorial: From Meristems to Floral Diversity: Developmental Options and Constraints
}

\author{
Regine Claßen-Bockhoff ${ }^{1 *}$, Louis Philippe Ronse De Craene ${ }^{2}$ and Annette Becker ${ }^{3}$ \\ ${ }^{1}$ Institute of Organismic and Molecular Evolution (iomE), Johannes Gutenberg-University, Mainz, Germany, ${ }^{2}$ Royal Botanical \\ Garden Edinburgh, Edinburgh, United Kingdom, ${ }^{3}$ Department of Biology, Institute of Botany, Justus-Liebig-University, \\ Gießen, Germany
}

Keywords: flower meristem, floral unit meristem, heterochrony, meristem expansion, spatial constraints, meristem identity

\section{Editorial on the Research Topic}

\section{From Meristems to Floral Diversity: Developmental Options and Constraints}

Meristems provide growth and shape in flowering plants. They are composed of undifferentiated cells whose fates depend on specific genetic and epigenetic processes. On the molecular level, differential gene expression, and hormone-mediated regulatory feedbacks play key roles, while on the morphogenetic level meristem geometry, mechanical pressure, meristem expansion and the timing of organ initiation and growth processes influence development. Combining the different aspects results in a deeper understanding of the developemental constraints and options underlying growth processes and the genesis of form in plant evolution.

\section{VEGETATIVE AND REPRODUCTIVE MERISTEMS}

\section{OPEN ACCESS}

Edited and reviewed by: Mark A. Elgar,

The University of Melbourne, Australia

*Correspondence: Regine Claßen-Bockhoff classenb@uni-mainz.de

Specialty section:

This article was submitted to Evolutionary Developmental Biology, a section of the journal Frontiers in Ecology and Evolution

Received: 04 December 2020 Accepted: 08 March 2021

Published: 31 March 2021

Citation:

Claßen-Bockhoff R, Ronse De Craene LP and Becker A (2021) Editorial: From Meristems to Floral Diversity: Developmental Options and Constraints.

Front. Ecol. Evol. 9:637954 doi: 10.3389/fevo.2021.637954
The shoot apical meristem (SAM) is the primary and dominant meristem maintaining open growth throughout the life of the plant. In the vegetative stage, it continuously segregates leaf primordia and lateral axial meristems. The reproductive stage involves the transition of some (or rarely all) SAMs into reproductive meristems (RMs). Whereas, inflorescence meristems (IMs) grow apically and segregate reproductive units, flower meristems (FMs), and floral unit meristems (FUMs) are determinate lacking apical growth (Claßen-Bockhoff and Bull-Hereñu, 2013).

Molecular studies in the model organism Arabidopsis thaliana (Brassicaceae) have shown that the activity of the SAM is regulated by a gene regulatory network mediated by the WUSCHEL/CLAVATA3 (WUS/CLA3) antagonistic system (Laux, 2003), whereas the FM is under the control of the FM identity genes LEAFY (LFY) and APETALA1 (AP1) (Chandler, 2012).

- Interestingly, some genes of the corresponding networks have specific functions whereas others have dual or multiple roles dependent on their respective partners. Chang et al. summarize the present knowledge of the genes with dual functions and show that e.g., WUS contributes to the maintenance of SAM activity, when associated with $C L V 3$, and to the termination of the FM in association with AGAMOUS (AG). The relative role of genes directly affects floral morphology. Obviously, deducing floral organ homology from single gene expressions may be misleading (Ochoterena et al., 2019).

- Lee et al. investigate the genetic interactions of the transcriptions factors (TF) CRABS CLAW (CRC) and SUPERMAN (SUP) and identify their target genes in Arabidopsis thaliana. These TFs not only play a key role in FM determinacy, but also regulate many genes during stamen development, even though CRC is only expressed in carpels and nectaries. The authors found that these TFs together influence meristem size, organ number, and stamen elongation, but also stimulate the expression of auxin- and cytokinin-related genes and fine-tune hormonal signaling. 
The study gives an impressive insight into the interactions between gene expression and epigenetic regulatory mechanisms.

The transition from indeterminate to determinate growth is of central interest for evolutionary developmental questions as it links organ formation with the two elementary features of open growth and reproduction. Meristem morphology, histology, developmental options, and gene regulatory processes completely change when the SAM merges into a FM, challenging the traditional interpretation of the flower as a short shoot with modified leaves (Bateman et al., 2006; Claßen-Bockhoff, 2016).

- In this context, the study of González et al. is of particular interest. The authors use the endoparasitic Pilostyles boyacensis (Apodanthaceae) to reconstruct the evolution of FM-related gene expression. This plant has no SAMs and a highly reduced plant body. Nevertheless, it produces flowers by a direct transition from the parenchyma strand to a FM. The authors compare gene expression at three different developmental stages and found that the lack of SAMs and leaves correlates with reductions or even absence in gene expression of specifically genes required for SAM maintenance.

If a SAM is not needed as a precondition for flower formation, why, then, should the FM be homologue to a SAM?

\section{DEVELOPMENTAL OPTIONS AND CONSTRAINTS IN FLOWER MERISTEMS}

Determinant meristems (FMs, FUMs) differ from SAMs and IMs by lacking apical growth. They do not segregate new primordia but fractionate until the meristem is completely used. This process can result in spatial constraints, organ suppression, and even organ reduction (Ronse de Craene, 2018). The first organs determine the space available to next organs. Spatial constraints are thus closely linked with the timing of floral organ initiation and development. On the evolutionary scale, heterochrony is one of the major drivers of morphological diversification through abbreviation (paedomorphosis) or extension of development (peramorphosis) (Box and Glover, 2010).

A second characteristic feature of determinate meristems is their ability to expand, thus compensating spatial constraints. Based on the auxin model (Reinhardt et al., 2003), primordia formation is a self-regulating process occupying space completely. Once additional space is generated, new primordia appear (Claßen-Bockhoff and Meyer, 2015) or existing primordia repeat fractionation (Ronse de Craene and Smets, 1991).

Altogether, flower development depends on spatio-temporal correlations in which available space and timing of primordium initiation influence each other and the symmetry, merism, and phyllotaxis of floral structures.

- As to floral symmetry, Naghiloo presents a comprehensive analysis across angiosperms comparing the onset of symmetry during ontogeny. Using published SEM data for early, mid, and late developmental stages, she found that both actinomorphic and zygomorphic patterns originate at different development stages and can be associated with changes and even reversals in symmetry. She concludes that floral symmetry is not necessarily a phylogenetic signal, but rather depends on epigenetic factors, such as mechanical pressure, space limitation, and meristem expansion.

- As to merism and phyllotaxis, Kitazawa and Fujimoto approach the Ranunculaceae as an appropriate model system to study the evolutionary relation between spiral and whorled organ position. They demonstrate that spiral and whorled phyllotaxis mainly depend on organ number and post-meristematic organ displacement. Spiral and whorled phyllotaxis are no alternatives but two sides from the same general law of size dependent growth rate, available space, and timing.

- Nuphar belongs to the basal angiosperms and has large bractless flowers occupying leaf positions in the parastichies of the rhizome. Floral organs are not clearly arranged in whorls or spirals. El et al. investigate flower position and floral organ initiation and conclude that the flowers are laterally arranged and the floral organization is primarily whorled. Irregularities are most likely caused by the relative proportions between flower meristem and floral organ primordia, mechanical pressure, and differences in timing.

- Bull-Hereñu and Ronse De Craene illustrate with the example of Malesherbia (Passifloraceae) that the diversity of flowers is correlated with the timing of organ initiation and the relative growth rate of the floral organs. While the different flower sizes are correlated with the FM size, the relative proportions between hypanthium and petal length depend on the timing of organ initiation and subsequent spatial constraints.

- Thaowetsuwan et al. investigate the origin of the perianth in the genus Croton (Euphorbiaceae) by comparing the development of staminate flowers with well-developed petals and carpellate flowers lacking petals or producing filamentous structures. The authors conclude that the filamentous structures are pedomorphic forms of petals. The reduction of petals is linked to spatial constraints as the alternipetalous whorl is initiated as the outer, but appears at anthesis as the inner whorl.

- Iwamoto et al. investigate the development of a fifth staminode in the labellum of Globbeae (Zingiberaceae). They demonstrate that some species have a well-developed or rudimentary fifth staminode, lacking in the majority of species, and conclude that the repeatedly appearing staminode might be a plesiomorphic character in the group. The loss of the fifth staminode may be linked with mechanical constrictions within the flower bud.

Meristem expansion is particularly obvious in FUMs. Once it exceeds a certain threshold, the meristem fractionates into submeristems.

- Claßen-Bockhoff and Frankenhäuser illustrate that the multistaminate unit in Ricinus communis (Euphorbiaceae) might be also interpreted as a floral unit. The structure, traditionally intepreted as a secondary polyandrous flower, develops from a large meristem which fractionates repetitively 
with increasing expansion. At the end, anthers associated with leaf-like structures are formed. As this developmental pattern is not known from any other polyandrous flower, the authors interpret the anthers as highly reduced staminate flowers of floral units matching the many monostaminate flowers present in the family.

\section{CONCLUSIONS}

The meristem level is often disregarded when morphogenetic processes are explained by developmental genetic studies and modeling approaches. However, gene expression and hormone flow depend on meristem conditions, which on their side are influenced by physical parameters. The Special Issue on Floral Meristems aims to increase the knowledge on meristem conditions and epigenetic processes to bridge the gap between developmental genetics, developmental morphology, and computer modeling. The papers on meristem conditions provide deep insights into the physical and genetic relations between vegetative and reproductive meristems. In particular, the multiple functions of genes and the formation of FMs in parasitic plants raise new questions on homology which need to be discussed in future. The papers on flower development clearly

\section{REFERENCES}

Bateman, R. M., Hilton, J., and Rudall, P. J. (2006). Morphological and molecular phylogenetic context of the angiosperms: contrasting the 'top-down' and 'bottom-up' approaches used to infer the likely characteristics of the first flowers. J. Exp. Bot. 57, 3471-3503. doi: 10.1093/jxb/erl128

Box, M. S., and Glover, B. J. (2010). A plant developmentalist's guide to paedomorphosis: reintroducing a classic concept to a new generation. Trends Plant Sci. 15, 241-246. doi: 10.1016/j.tplants.2010.02.004

Bull-Hereñu, K., Claßen-Bockhoff, R., and Ronse De Craene, L. P. (2014). The FLO-RE-S network for contemporary studies in flower structure and biology. Flora 221, 1-3. doi: 10.1016/j.flora.2016.02.005

Chandler, J. W. (2012). Floral meristem initiation and emergence in plants. Cell. Mol. Life Sci. 69, 3807-3818. doi: 10.1007/s00018-012-0999-0

Claßen-Bockhoff, R. (2016). The shoot concept of the flower: still up to date? Flora 221, 46-53. doi: 10.1016/j.flora.2015.11.012

Claßen-Bockhoff, R., and Bull-Hereñu, K. (2013). Towards an ontogenetic understanding of inflorescence diversity. Ann. Bot. 112, 1523-1542. doi: 10.1093/aob/mct009

Claßen-Bockhoff, R., and Meyer, C. (2015). Space matters: meristem expansion triggers corona formation in Passiflora. Ann. Bot. 117, 277-290. doi: $10.1093 / \mathrm{aob} / \mathrm{mcv} 177$

Iwamoto, A., and Bull-Hereñu, K. (2018). Floral development: re-evaluation of its importance. J. Plant Res. 131, 365-366 doi: 10.1007/s10265-018-1034-9

Laux, T. (2003). The stem cell concept in plants: a matter of debate. Cell 113, 281-283. doi: 10.1016/S0092-8674(03)00312-X illustrate the effects of growth dynamics, spatial constraints, mechanical pressure, meristem expansion, and novel space generation on the number, size, and shape of floral organs. They underline the need to consider epigenetic processes when dealing with character transformation, heterotopy, and heterochrony on an evolutionary scale.

\section{AUTHOR CONTRIBUTIONS}

All authors listed have made a substantial, direct and intellectual contribution to the work, and approved it for publication.

\section{ACKNOWLEDGMENTS}

This is the third Special Issue co-edited by members of the FLORE-S group (Bull-Hereñu et al., 2014; Iwamoto and Bull-Hereñu, 2018). FLO-RE-S aims to increase interest in organismic aspects of floral development ranging from meristem conditions to biotic interactions (https://flores-network.com). We are grateful for the support of the entire editorial board at Frontiers in Ecology and Evolution. Thanks go also to the external peer reviewers who gave their time and insights to enhance the quality of the manuscripts in this issue.
Ochoterena, H., Vrijdaghs, A., Smets, E., and Claßen-Bockjoff, R. (2019). The search for common origin: homology revisited. Syst. Biol. 68, 767-780. doi: $10.1093 /$ sysbio/syz013

Reinhardt, D., Pesce, E. R., Stieger, P., Mandel, T., Baltensperger, K., Bennett, M., et al. (2003). Regulation of phyllotaxis by polar auxin transport. Nature 426, 255-260. doi: 10.1038/nature02081

Ronse de Craene, L. P. (2018). Understanding the role of floral development in the evolution of angiosperm flowers: clarifications from a historical and physicodynamic perspective. J. Plant Res. 1313, 367-393. doi: 10.1007/s10265-018-1021-1

Ronse de Craene, L. P., and Smets, E. F. (1991). The impact of receptacular growth on polyandry in the myrtales. Bot. J. Linn. Soc. 105, 257-269 doi: 10.1111/j.1095-8339.1991.tb0 0207.x

Conflict of Interest: The authors declare that the research was conducted in the absence of any commercial or financial relationships that could be construed as a potential conflict of interest.

Copyright (c) 2021 Claßen-Bockhoff, Ronse De Craene and Becker. This is an openaccess article distributed under the terms of the Creative Commons Attribution License (CC BY). The use, distribution or reproduction in other forums is permitted, provided the original author(s) and the copyright owner(s) are credited and that the original publication in this journal is cited, in accordance with accepted academic practice. No use, distribution or reproduction is permitted which does not comply with these terms. 